Araştırma ve Geliştirme Dergisi

International Journal of

Engineering Research and

Development
UMAGD, (2021) 13(2), 478-488.

10.29137/umagd.859150

Cilt/Volume:13 Sayı/Issue:2 Haziran/June 2021

Araștırma Makalesi / Research Article

\title{
OFDM-AIM İletim Tekniği için Karınca Kolonisi Optimizasyon Algoritması Tabanlı Alt-Blok Set Tasarımı
}

\author{
Ant Colony Optimization Algorithm Based Sub-Blocks Set Design for OFDM-AIM \\ Transmission Technique
}

\author{
Mahmut Yıldırım1 iD \\ ${ }^{1}$ Bozok Üniversitesi, Elektrik Elektronik Mühendisliği Bölümü, 66900 Yozgat, TÜRKIYYE
}

Başvuru/Received: 12/01/2021Ｋabul/Accepted: 18/03/2021Ｃ̧evrimiçi Basım / Published Online: 27/05/2021

Son Versiyon/Final Version: 18/06/2021

\section{$\ddot{O} \mathbf{z}$}

OFDM-IM (İndeks Modülasyonlu Dikgen Frekans Bölmeli Çoğullama), 5G (Beşinci Nesil) ötesi iletişim sistemleri için aday iletim tekniklerinden birisi olarak son yıllarda üzerinde birçok çalışma gerçekleştirilmiştir. OFDM-IM'nin spektral verimliliğinin artırılması amacıyla geliştirilen OFDM-AIM (Tüm İndeks Modülasyonlu OFDM) tekniğinde, boş alt-taşıyıcıların bulunduğu OFDM-IM'den farklı olarak tüm alt-taşıııcılar aktif duruma getirilmiştir. Bu makalede, OFDM-AIM'in bit hata oranı (BHO) performansının iyileştirilmesi için Karınca Kolonisi Optimizasyonu (KKO) algoritması tabanlı bir alt-blok set tasarımı önerilmiştir (KKO-OFDM-AIM). OFDM-AIM tekniğinde veri bitleri, PSK/QAM takımyıldızı noktaları ile eşleştirilirken önerilen KKOOFDM-AIM tekniğinde, çeşitlilik kazancının artırılması amacıyla PSK/QAM takımyıldızı uzayının tamamı olası eşleştirme noktaları olarak değerlendirilir. KKO-OFDM-AIM tekniğinde, en yüksek uygunluk değerine sahip eşleştirme noktaları KKO algoritması tarafından belirlenerek alt-blok seti oluşturulur. Simülasyon çalışmalarında KKO-OFDM-AIM tekniği, OFDM, OFDMIM, OFDM-AIM ve GA-OFDM-AIM (Genetik algoritma tabanlı OFDM-AIM) teknikleri ile karşılaştırılmış ve en iyi BHO başarısına sahip olduğu görülmüştür.

\section{Anahtar Kelimeler}

“5G, OFDM-IM, OFDM-AIM, Karınca Kolonisi Optimizasyonu”

\begin{abstract}
OFDM-IM (Orthogonal Frequency Division Multiplexing with Index Modulation) as one of the candidate transmission techniques for communication systems beyond 5G (Fifth Generation), many studies have been carried out in recent yeaars. In the OFDM-AIM (OFDM with All Index Modulation) technique, which has developed to increase the spectral efficiency of the OFDM-IM, unlike the OFDM-IM which has empty sub-carriers, all sub-carriers are activated. In this article, Ant Colony Optimization (ACO) algorithm based sub-block set design (ACO-OFDM-AIM) is proposed to improve bit error rate (BER) performance of the OFDM-AIM. In the OFDM-AIM technique, data bits are matched with PSK/QAM constellation points, while in the KKO-OFDM-AIM technique, the entire PSK/QAM constellation space can be determined as possible mapping points in order to increase diversity gain. In the ACOOFDM-AIM technique, the matching points with the highest fitness value are determined by the ACO algorithm and a sub-block set is created. In simulation studies, the ACO-OFDM-AIM technique is compared with OFDM, OFDM-IM, OFDM-AIM, and GAOFDM-AIM (Genetic algorithm-based OFDM-AIM) techniques and it is seen that ACO-OFDM-AIM has the best BER performance.
\end{abstract}

Key Words

"5G, OFDM-IM, OFDM-AIM, Ant Colony Optimization" 


\section{Giriş}

MIMO (Çoklu giriş çoklu çıkış, Multiple input multiple Output) anten tekniği (Lu vd., 2014), günümüz iletişim sistemlerinde yaygın bir şekilde kullanılıyor olmasının yanı sıra 5G (beşinci nesil, Fifth Generation) (Andrews vd., 2014) ve ötesi kablosuz iletişim sistemleri için de temel teknolojiler arasında yer almaktadır. Bununla birlikte antenler-arası eşleme (inter-antenna synchronization) ve kanallararası girişim (inter-channel interference) problemleri, MIMO tekniğini kullanan sistemlerin performansını sınırlamaktadır. MIMO'nun bu dezavantajının aşılması amacıyla bir anten dizisi içerisinde aktif duruma getirilecek antenlerin (anten indeksleri) veri bitleri kullanılarak belirlenmesi prensibine dayanan SM (uzaysal modülasyon, Spatial Modulation) tekniği önerilmiştir (Wen vd., 2019). SM'nin yapısı sayesinde veri bitlerinin bir bölümünün anten indeksleri ile taşınarak spektral verimliliğin (spectral efficiency) artırılması sağlanmiştır.

MIMO tekniği gibi OFDM (Dikgen Frekans Bölmeli Çoğullama, Orthogonal Frequency Division Multiplexing) tekniği de günümüz kablosuz iletişim sistemlerinde yaygın olarak kullanılmaktadır (Hwang vd., 2009; Boroujeny \& Moradi, 2016). Bu sistemler arasında WiMAX (Mikrodalga Erişimi için Dünya Çapında Uyumluluk, Worldwide Interoperability for Microwave Access (IEEE 802.16)), LTE (Uzun Vadeli Evrim, Long Term Evolution) ve WLAN (Kablosuz Yerel Alan Ağları, Wireless Local Area Networks (IEEE 802.11)) vb. yer almaktadır (Andrews vd., 2007; Dahlman vd., 2013; Crow vd., 1997). Ayrıca, 5G ve ötesi iletişim sistemleri için aday iletim tekniklerinin büyük ölçüde OFDM temel alınarak geliştirilmesi, OFDM üzerindeki geniş çaplı çalışmaların sürdürülmesine neden olmaktadır. Bu çalışmalar arasında SM tekniğinden esinlenerek geliştirilen OFDM-IM (İndeks Modülasyonlu Dikgen Frekans Bölmeli Çoğullama, Orthogonal Frequency Division Multiplexing with Index Modulation), tekniği önemli bir yere sahiptir (Başar vd., 2013).

OFDM-IM tekniğinde mesaj bit bloğu, veri bitleri ve indeks bitleri olmak üzere iki gruba ayrılır. İndeks bitleri, aktif ya da pasif duruma getirilecek alt-taşıyıcıları belirleyerek alt-taşıyıcı indekslerini oluşturur. Alt-taşıyıcı indeksleri yoluyla indeks bitlerinin iletilmesi sağlanır. Veri bitleri ise PSK/QAM takımyıldızı eşleştirilmeleri yapılarak aktif alt-taşıyıcılar tarafından iletimi gerçekleştirilir. OFDMIM'nin bu yapısı tüm alt-taşıyıcıları aktif olan OFDM'den temel farkını ortaya koymaktadır. OFDM-IM sahip olduğu düşük PAPR (tepe gücü ortalama güç oranı, Peak to Average Power Ratio), daha iyi bir BER (Bit Hata Oranı, Bit Error Rate) performansı, enerji verimliliği (energy efficiency) ve kanallar-arası girişime karşı dayanıklılık gibi özellikleri OFDM’ye göre üstünlükleri olarak sıralanabilir (Başar vd., 2013; Wen vd., 2017; Başar 2016; Fan vd., 2015).

Yukarıda sıralanan avantajlarının yanı sıra, kullanılmayan alt-taşıyıcıların varlığı OFDM-IM'nin performansını sınırlayan en büyük etkenlerden birisidir. Bu dezavantajın giderilmesi amacıyla literatürde son yıllarda birçok çalışma gerçekleştirilmiştir (Başar, 2015; Xiao vd., 2014; Mao vd., 2017; Shi vd., 2019). Başar (2015) çalışmasında, uzay-zaman blok kodları ve koordinat serpiştirme OFDMIM tekniği ile birleştirilerek (CI-OFDM-IM (Koordinat Serpiştirme OFDM-IM, Coordinate Interleaving OFDM-IM) çeşitlilik kazancının artırılmasını sağlamış ve bu yolla sistemin BER başarısını artırmıştır. Çeşitlilik kazancı, alt-taşıyıcıların birbirlerine mümkün olduğu kadar benzememesinin bir ölçüsü olarak ifade edilebilir. Xiao vd. (2014) çalışmalarında, en uygun aktif alt-taşıyıcı sayısını, sistemin BER performansının artırılması amacıyla araştırmışlardır. OFDM-IM'nin boş alt taşıyıcılara sahip olmasından kaynaklı nispeten düşük spektral verimliliğinin artırılması amacıyla DM-OFDM-IM (çift modlu OFDM-IM, Dual Mode OFDM-IM) önerilmiştir (Mao vd., 2017). DM-OFDM-IM'de alt-taşıyıcıların tamamı aktif edilirken mesaj bit bloğu, OFDM-IM'de olduğu gibi veri bitlerine ve indeks bitlerine bölünür. İndeks bitleri, iki farklı uzaya ayrılan PSK/QAM takımyıldızı seçimini gerçekleştirirken, veri bitleri ise tamamı aktif olan alt-taşıyıcılar aracılığıla iletilirler.

OFDM-IM temel alınarak geliştirilen ve alt-taşıyıcıların tamamının kullanıldığı OFDM-AIM (Tüm İndeks Modülasyonlu OFDM, OFDM with All Index Modulation) tekniğinde, çeşitlilik kazancının artırılmasıyla birlikte BER performansının iyileştirilmesi sağlanmıştır (Shi vd., 2019). Genetik algoritma (Genetic Algorithm) tabanlı bir OFDM-AIM tekniğini (Shi vd. 2019) önererek (GAOFDM-AIM) çeşitlilik kazancının daha da artırılmasını hedeflenmişlerdir. OFDM-AIM tekniğinde sabit olan PSK/QAM takımyıldızı noktaları, genetik algoritma yardımıyla çeşitlilik kazancının artıılması amacıyla çözüm uzayında farklı noktalar aranarak optimize edilir. En iyi BER değerine sahip yeni takımyıldızı noktaları optimum alt-blok seti olarak belirlenir. OFDM-AIM tabanlı başka bir altblok set tasarımı ise Yıldırım (2020) tarafından ABC (yapay arı kolonisi, Artificial Bee Colony) algoritması kullanılarak gerçekleştirilmiştir.

Bu çalışmada, OFDM-AIM tekniğinin çeşitlilik kazancının artırılarak BER performansının iyileştirilmesi amacıyla KKO (Karınca Kolonisi Optimizasyonu, Ant Colony Optimization) algoritması (Chen vd., 2013) tabanlı alt-blok set tasarımı önerilmiştir (KKOOFDM-AIM). KKO algoritması ile alt-blok set tasarımında, optimum alt-taşıyıcı noktaları PSK/QAM takımyıldızı uzayında araştırılmıştır. KKO optimizasyonunda alt-blok setlerinin uygunlukları, iletişim sisteminin ABEP (Ortalama Bit Hata Olasılığı, Average Bit Error Probability) değerine göre belirlenmektedir. Önerilen KKO-OFDM-AIM'nin performansı, OFDM, OFDM-IM, OFDM-AIM ve GA-OFDM-AIM ile BER başarı kriterine göre karşılaştırılmış ve en iyi sonucu veren teknik olduğu görülmüştür. Ayrıca KKO algoritması ile alt-blok set tasarımı, çevrim dışı bir optimizasyon süreci olduğu için sistemin iletim hızına olumsuz bir etkisi bulunmamaktadır. 
Makalenin geri kalan bölümleri şu şekilde düzenlenmiştir: 2. bölümde, OFDM, OFDM-IM ve OFDM-AIM iletim teknikleri, 3 . bölümde, OFDM-AIM için önerilen karınca kolonisi optimizasyon algoritması tabanlı alt-blok set tasarımı, 4. bölümde, simülasyon çalışmaları ve 5. bölümde sonuçlar verilmiştir.

\section{2. İletim Teknikleri}

\subsection{OFDM}

Günümüz iletişim sistemlerinde en çok tercih edilen çoğullama tekniklerinden birisi olan OFDM ile birlikte semboller-arası girişim (Inter-sysmbol Interference) ve kanallar-arası girişim problemleri önemli ölçüde azaltılmıştır. MIMO teknolojisini kullanan iletişim sistemlerinde de sıklıkla kullanılan OFDM'nin temel blok şeması Şekil 1'de görülmektedir.

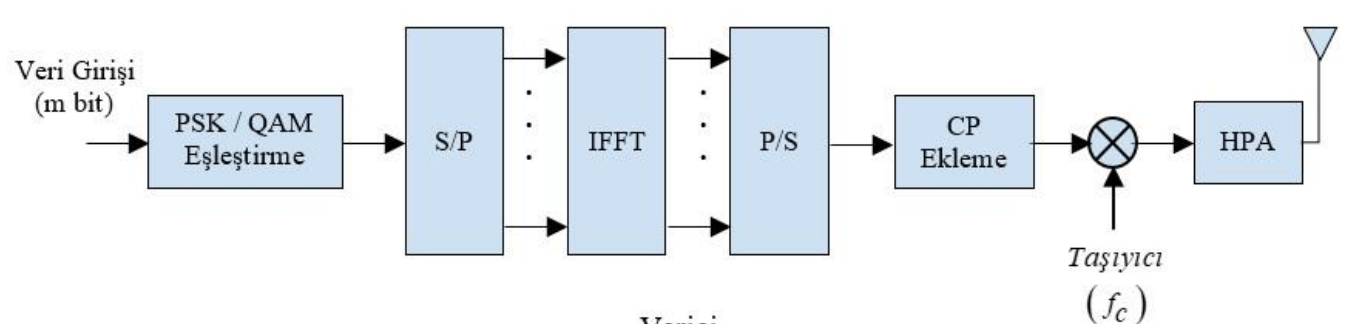

Verici

$\left(f_{c}\right)$

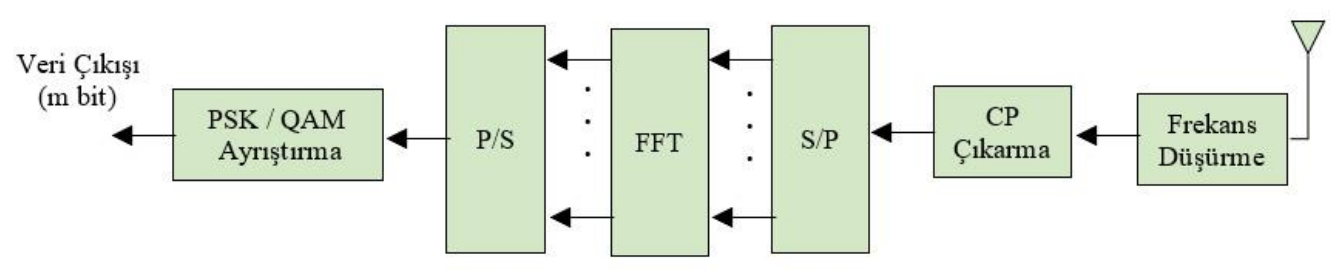

Alıcı

Şekil 1. OFDM iletim tekniğinin blok diyagramı.

İlk olarak, Mesaj bitleri ile PSK/QAM takımyıldızı eşleştirmeleri yapılır. Bu sayede, haberleşme sistemi için tahsis edilen bant genişliğinin daha verimli kullanılması sağlanmış olur. Daha sonra, S/P (Seri/Paralel dönüşüm) ve IFFT (Ters H1zlı Fourier Dönüşümü, Inverse Fast Fourier Transform) işlemleri gerçekleştirilir. IFFT işlemi ile birlikte $N$ adet alt-taşıyıcı üretilirken işaretlerin de frekans ekseninden zaman eksenine aktarımı sağlanmış olur. $N$ IFFT uzunluğuna sahip bir OFDM sembolü $\boldsymbol{x}=\left[x_{1}, x_{1}, \cdots, x_{N-1}, x_{N}\right]$ şeklinde ifade edilebilir. P/S (Paralel/Seri dönüşüm) ardından bir sonraki adım olan CP (Döngüsel Önek, Cycle Prefix) bloğu ile semboller-arası girişimin önlenmesi hedeflenmektedir. Döngüsel önek işlemi, $N$ uzunluğundaki bir OFDM sembolü dizisindeki son $N / 8$ veya $N / 4$ uzunluğundaki $\left(L_{C P}\right)$ bölümünün aynı dizinin önüne eklenmesi ile gerçekleştirilmektedir. Böylece bir OFDM sembolü, $N+L_{C P}$ uzunluğa sahip olmaktadır. Yüksek frekanslı taşıyıcı işareti ile frekansı yükseltilen OFDM sembolü son olarak, HPA (Yüksek Güç Kuvvetlendirici, High Power Amplifier) ile kuvvetlendirilerek iletim kanalına aktarılır.

İletim kanalından geçerek sistem alıcı birimine ulaşan mesaj işareti, ilk olarak frekans düşürme işlemi ile temel bant frekans seviyesine indirgenir. Döngüsel önek bilgisi çıkarılan işaret, Seri/Paralel dönüşümü yapıldıktan sonra, FFT işlemi ile birlikte zaman ekseninden frekans eksenine aktarılmış olur. Paralel/Seri dönüşümden sonra PSK/QAM takımyıldızı eşleştirmeleri çözülerek mesaj bitleri elde edilir. Veri girişindeki mesaj bitleri ile sistemin alıcı biriminden sonunda elde edilen mesaj bitleri karşılaştırılarak sistemin BER performansı belirlenmektedir.

OFDM temelli iletim tekniklerinde önemli bir parametre olan SE (Spektral Verimlilik, Spectral Efficiency), herhangi bir iletim tekniğinde $N+L_{C P}$ uzunluğundaki bir sembolde iletilen bit sayısı ile orantılıdır.

$S E=\frac{m}{N+L_{C P}}[b i t / s / H z]$

burada $m$ bir sembolde iletilen toplam bit sayısı, $s$ saniye ve $H z$ frekans birimleridir.

\subsection{OFDM-IM}

$5 \mathrm{G}$ ve ötesi iletişim sistemleri için aday iletim tekniklerinden birisi olarak son y1llarda üzerinde birçok çalışma gerçekleştirilen OFDMIM'nin blok şeması Şekil 2'de görülmektedir. OFDM-IM sembolü, her birinde $n$ adet alt-taşıyıcı bulunan $G$ adet alt-bloğun birleşmesiyle toplamda $N$ adet alt-taşıyıcıdan oluşmaktadır. Bir OFDM-IM sembolünde iletilecek olan $m$ adet mesaj bit bloğu, bit 

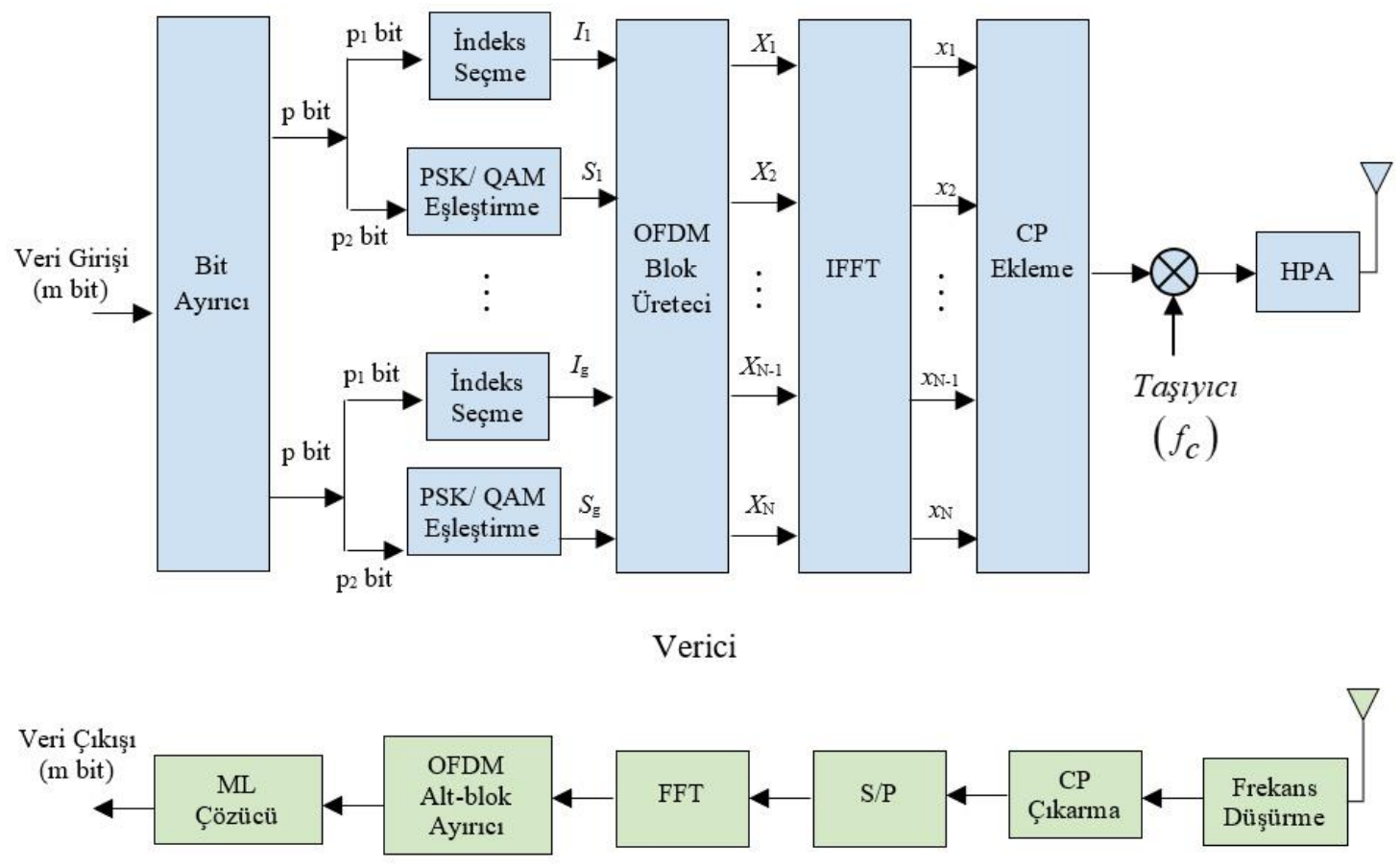

Alic1

Şekil 2. OFDM-IM iletim tekniğinin blok diyagramı.

Tablo 1. OFDM-IM için alt-taşıyıcı şablonu $(n=4)$

\begin{tabular}{ccc}
\hline İndeks bitleri $\left(\boldsymbol{p}_{\mathbf{1}}\right)$ & Alt-taşıyıcı İndeks & Alt-taşıyıcılar \\
\hline$\left[\begin{array}{lll}0 & 0\end{array}\right]$ & {$[1,2]$} & {$\left[\mathrm{S}_{\mathrm{A} 1}, \mathrm{~S}_{\mathrm{A} 2}, \mathrm{~S}_{\mathrm{P} 1}, \mathrm{~S}_{\mathrm{P} 2}\right]$} \\
{$\left[\begin{array}{ll}0 & 1\end{array}\right]$} & {$[2,3]$} & {$\left[\mathrm{S}_{\mathrm{P} 1}, \mathrm{~S}_{\mathrm{A} 1}, \mathrm{~S}_{\mathrm{A} 2}, \mathrm{~S}_{\mathrm{P} 2}\right]$} \\
{$\left[\begin{array}{ll}1 & 0\end{array}\right]$} & {$[3,4]$} & {$\left[\mathrm{S}_{\mathrm{P} 1}, \mathrm{~S}_{\mathrm{P} 1}, \mathrm{~S}_{\mathrm{A} 2}, \mathrm{~S}_{\mathrm{A} 2}\right]$} \\
{$\left[\begin{array}{ll}1 & 1\end{array}\right]$} & {$[1,4]$} & {$\left[\mathrm{S}_{\mathrm{A} 1}, \mathrm{~S}_{\mathrm{P} 1}, \mathrm{~S}_{\mathrm{P} 2}, \mathrm{~S}_{\mathrm{A} 2}\right]$} \\
\hline
\end{tabular}

ayırıcı tarafindan her birinde $p$ bit olmak üzere $G$ gruba ayrılır $(m=G * p)$. $p$ adet bit, indeks bitleri $\left(p_{1}\right)$ ve veri bitleri $\left(p_{2}\right)$ olmak üzere iki gruba ayrılır. İndeks bitleri, bir alt-bloktaki aktif ya da pasif edilecek alt-taşıyıcıları belirler. Tablo 1'de alt-taşıyıcı sayısı $n=$ 4 , aktif alt-taşıyıcı sayısı $k=2$ ve pasif alt-taşıyıcı sayısı $l=2$ değerlerine sahip bir alt-blok yapısı görülmektedir. 2 bitlik indeks bit dizisinin olası değerlerine göre oluşturulan toplam $2^{p_{1}}=2^{2}=4$ alt-blok için $S_{A}$ ve $S_{P}$ sirasılyla aktif ve pasif alt-taşıyıcıları temsil etmektedir. Böylelikle, aktif ve pasif alt-taşıyıcıların konumu ile indeks bitleri iletilebilmektedir. Veri bitleri, PSK/QAM takımyıldızı eşleştirmeleri yapılarak aktif durumda olan alt-taşıyıcılar yoluyla iletilir. Üretilen $G$ adet alt-blok, OFDM blok üreteci ile tekrar $N$ adet alt-taşıyıcı dizisi şeklinde birleştirilir. Daha sonra sırasıyla IFFT, döngüsel önek ekleme, frekans yükseltme ve işaret kuvvetlendirme işlemleri ile birlikte OFDM-IM sembolleri iletişim kanalına aktarılır. Sistemim alıcı birimine ulaşan ve sırasıyla frekans düşürme, $\mathrm{CP}$ çıarma, S/P dönüştürme ve FFT işlemleri uygulanan $N$ uzunluğa sahip işaret dizisi, aşağıdaki gibi ifade edilebilir:

$\mathbf{y}=\mathbf{X h}+\mathbf{w}$

Burada $\mathbf{X}$, sistem verici birimindeki OFDM blok üreteci tarafından oluşturulan işaret dizisi, $\mathbf{h}$ kanal vektörü ve $\mathbf{w}$ kanal gürültü vektörüdür. Daha sonra, y OFDM alt-blok ayırıcı tarafından $G$ adet alt-bloka dönüştürülür. Dönüşüm sonrasında, her bir alt-blok aşağıdaki gibi temsil edilebilir:

$\mathbf{y}_{g}=\mathbf{S}_{g} \mathbf{h}_{g}+\mathbf{w}_{g}, \quad g=1, \cdots, G$

Burada $\mathbf{S}_{\boldsymbol{g}}$ sistem verici biriminde oluşturulan $G$ adet alt-bloktan her birini temsil etmektedir. Son olarak, mesaj bit bloğu ve vericide üretilen alt-bloklar, ML (Maksimum Olabilirlik Maximum Likelihood) işlemi ile tespit edilmeye çalışılır.

$\widehat{\mathbf{S}_{g}}=\arg \min _{\mathbf{S}_{g}}\left\|\mathbf{y}_{g}-\mathbf{S}_{g} \mathbf{h}_{g}\right\|^{2}$ 
Burada $\|\cdot\|$, Öklid normunu, arg $\underset{\mathbf{s}_{\boldsymbol{g}}}{\min }\|\cdot\|$ ise öklid normunun minimum değerini temsi etmektedir.

\subsection{OFDM-AIM}

OFDM-IM tekniğinden esinlenerek geliştirilen OFDM-AIM tekniği, alt-taşıyıcılarının tümünün aktif olması sebebiyle OFDM-IM'ye göre daha fazla çeşitlilik kazancı sunmaktadır. Şekil 3'de görüldüğü üzere $N$ uzunluğundaki bir OFDM-AIM sembolü, her birinde $n$ adet alt-taşıyıcı bulunan $G$ adet alt-bloktan oluşmaktadır. OFDM-AIM sembolü üretiminde ilk olarak, $m$ adet mesaj bit bloğu, bit ayırıcı tarafından $p$ uzunluğundaki veri bit dizilerine ayrılır. Veri bitlerinin PSK/QAM takımyıldızı eşleştirilmeleri gerçekleştirildikten sonra, $n$ adet çoğullanan her bir eşleştirme bir alt-taşıyıcı tarafından iletilir.

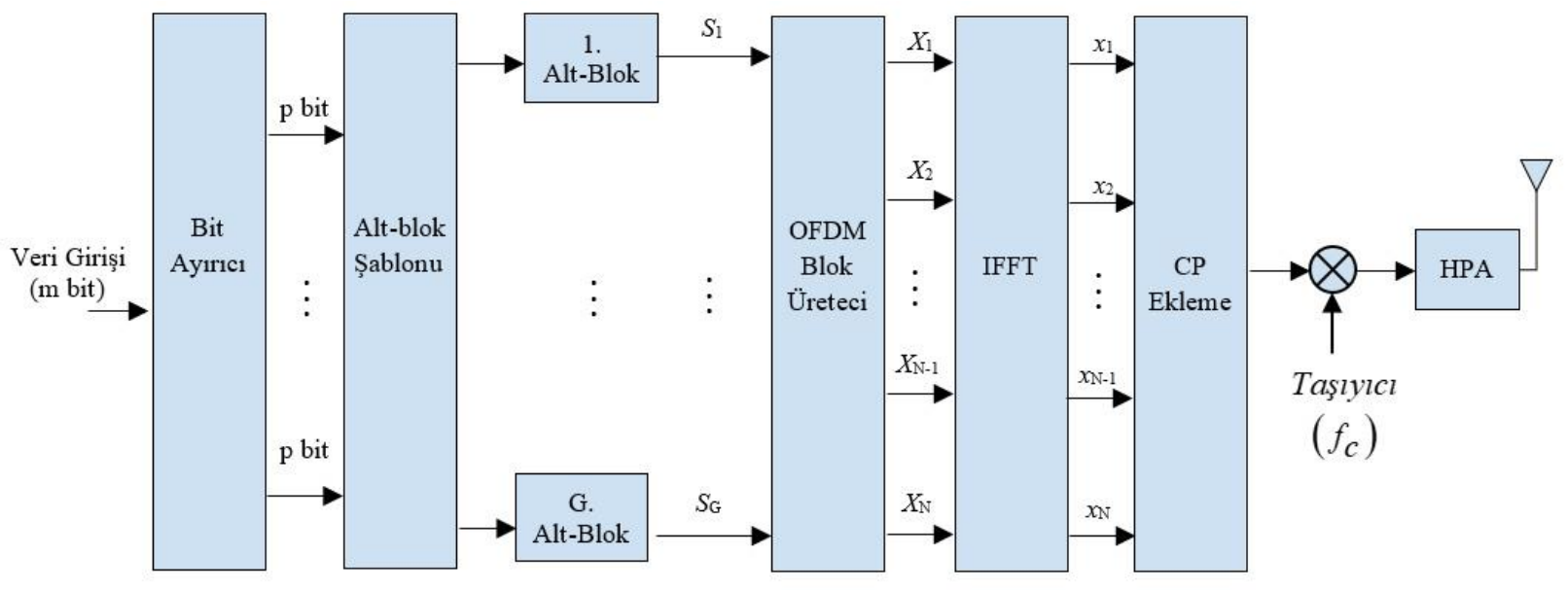

Verici

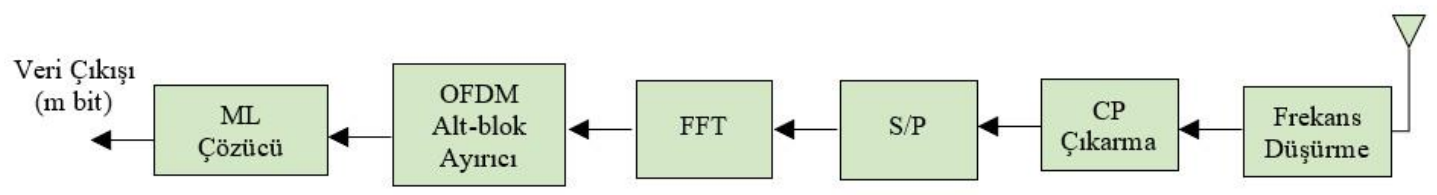

Alic1

Şekil 3. OFDM-AIM iletim tekniğinin blok diyagramı.

Tablo 2'de alt-taşıyıcı sayısı $n=4$, veri bit sayısı $p=4$ değerlerine sahip bir alt-blok şablonu görülmektedir. Alt-blok şablonuna göre üretilen $G$ adet alt-blok, OFDM blok üreteci ile tekrar $N$ adet alt-taşıyıcı dizisi şeklinde birleştirildikten sonra sırasıyla IFFT, döngüsel önek ekleme, frekans yükseltme ve işaret kuvvetlendirme işlemleri ile birlikte OFDM-AIM sembolleri elde edilmiş olur. Şekil 3'de görüldüğ̈̈ gibi OFDM-AIM tekniği, OFDM-IM'den alt-blok üretme işleminde farklılık gösterirken diğer alt işlemlerde benzer bir yaprya sahiptir.

Tablo 2. OFDM-AIM için alt-taşıyıcı şablonu $(n=4)$

\begin{tabular}{|c|c|}
\hline Veri bitleri $(p)$ & Alt-taşıyıcılar \\
\hline$\left[\begin{array}{llll}0 & 0 & 0 & 0\end{array}\right]$ & {$\left[\mathrm{S}_{\mathrm{A} 1}, \mathrm{~S}_{\mathrm{A} 1}, \mathrm{~S}_{\mathrm{A} 1}, \mathrm{~S}_{\mathrm{A} 1}\right]$} \\
\hline$\left[\begin{array}{llll}0 & 0 & 0 & 1\end{array}\right]$ & {$\left[\mathrm{S}_{\mathrm{A} 2}, \mathrm{~S}_{\mathrm{A} 2}, \mathrm{~S}_{\mathrm{A} 2}, \mathrm{~S}_{\mathrm{A} 2}\right]$} \\
\hline$\cdots$ & \\
\hline$\left[\begin{array}{llll}1 & 1 & 1 & 0\end{array}\right]$ & {$\left[\mathrm{S}_{\mathrm{A} 15}, \mathrm{~S}_{\mathrm{A} 15}, \mathrm{~S}_{\mathrm{A} 15}, \mathrm{~S}_{\mathrm{A} 15}\right]$} \\
\hline$\left[\begin{array}{llll}1 & 1 & 1 & 1\end{array}\right]$ & {$\left[\mathrm{S}_{\mathrm{A} 16}, \mathrm{~S}_{\mathrm{A} 16}, \mathrm{~S}_{\mathrm{A} 16}, \mathrm{~S}_{\mathrm{A} 16}\right]$} \\
\hline
\end{tabular}

\section{3. Önerilen KKO Algoritması Tabanlı OFDM-AIM Tekniği (KKO-OFDM-AIM)}

KKO algoritması, gerçek karınca kolonilerinin yiyecek arama davranışlarının modellenmesiyle geliştirilmiş popülasyon tabanlı bir yapay zeka algoritmasıdır. Karıncalar, bir yiyecek kaynă̆ araştırırken feromon adlı bir sıvı salgılarlar. Zamanla buharlaşıp yok olma özelliği gösteren Feromon, bir yiyecek kaynağının, yuvadan uzaklığı (uygunluk değeri) hakkında kolonideki diğer karıncalara bilgi 


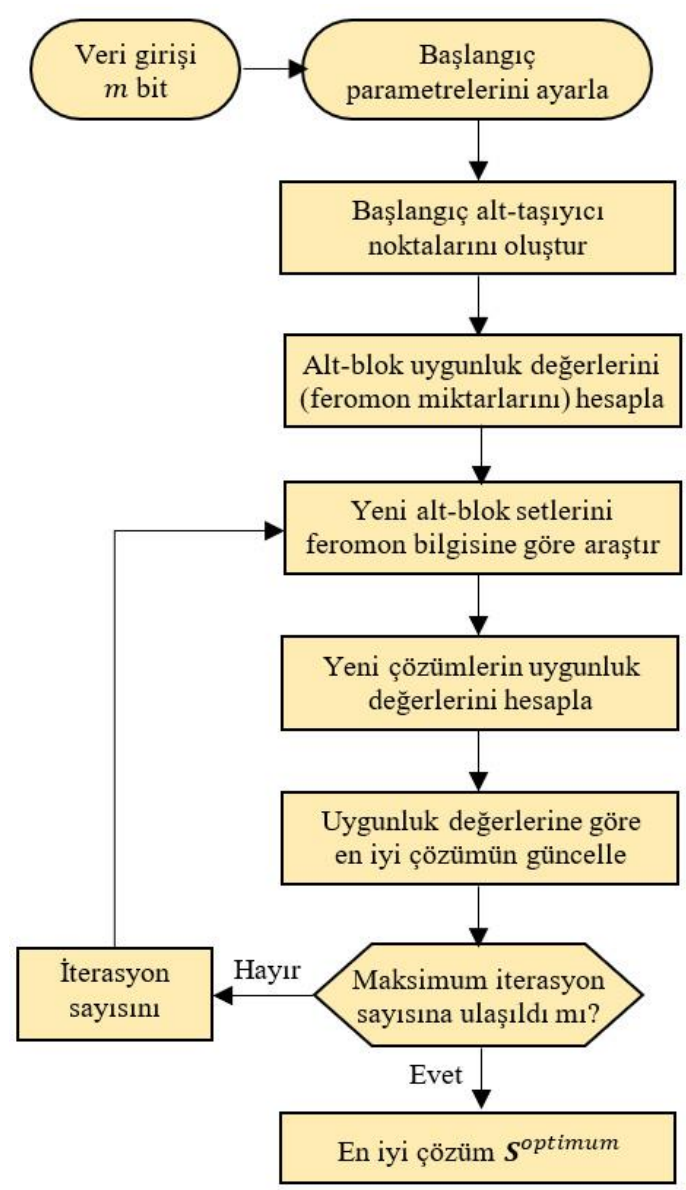

Şekil 4. Karınca Kolonisi Optimizasyonu (KKO) Algoritması akış şeması.

sağlamaktadır. Feromon yoğunluğunun çok olduğu güzergahtaki yiyecek kaynağının yuvaya daha yakın (yüksek uygunluk değeri), feromon yoğunluğunun az olduğu güzergahtaki yiyecek kaynağının ise yuvaya uzak olduğu (düşük uygunluk değeri) anlamına gelmektedir. Şekil 4'de KKO algoritması ile alt-blok set tasarımının akış şeması görülmektedir.

KKO ile alt-blok set tasarımında, her bir yiyecek kaynă̆ının rotası bir alt-blok setini, ilgili alt-blok setinin feromon miktarı yani uygunluğu ise ABEP (Ortalama Bit Hata Olasılığı, Average Bit Error Probability) değerini temsil etmektedir. Bir OFDM-AIM iletim tekniğinin ABEP değeri aşağıdaki gibi hesaplanabilir (Başar vd., 2013):

$P_{A B E P}=\frac{1}{p n_{S_{g}}} \sum_{S_{g}} \sum_{\hat{S}_{g}} P_{U}\left(S_{g} \rightarrow \hat{S}_{g}\right) \epsilon\left(S_{g}, \hat{S}_{g}\right)$

burada $\epsilon\left(S_{g}, \hat{S}_{g}\right)$ vericide üretilen $S_{g}$ alt-bloğunun alıcıda hatalı çözülmesi $\left(\hat{S}_{g}\right)$ durumundaki hatalı bit sayısını, $n_{S_{g}}=2^{p}$ olası tüm $S_{g}$ alt-blok sayısını, $P_{U}\left(S_{g} \rightarrow \hat{S}_{g}\right)$ aşağıda gösterildiği üzere koşulsuz UPEP’i (Koşulsuz İkili Hata Olasılığını, Unconditional Pairwise Error Probability) ifade etmektedir (Başar vd., 2013).

$P_{U}\left(\mathbf{S}_{g} \rightarrow \widehat{\mathbf{S}}_{g}\right) \approx \frac{1 / 12}{\operatorname{det}\left(\mathbf{I}_{n}+A_{n} \boldsymbol{\gamma} / 4 N_{0, F}\right)}+\frac{1 / 12}{\operatorname{det}\left(\mathbf{I}_{n}+\mathbf{A}_{n} \boldsymbol{\gamma} / 4 N_{0, F}\right)}$

burada $\mathbf{I}_{n}$ ve $A_{n}$, sirasiyla $n \times n$ boyutlarında birim matris ve korelasyon matrisi, $\boldsymbol{\gamma}=\left(S_{g}-\hat{S}_{g}\right)^{H}\left(S_{g}-\hat{S}_{g}\right),(\cdot)^{H}$ Hermitian dönüşümünü ifade etmektedir.

OFDM-AIM tekniğinde veri bitleri, reel ve sanal eksendeki değerleri sabit olan PSK/QAM takımyıldızı noktaları ile eşleştirilirken KKO-OFDM-AIM tekniğinde, çeşitlilik kazancının daha da artırılması amacıyla PSK/QAM takımyıldızı uzayının tamamı olası eşleştirme noktaları olarak belirlenebilir. KKO algoritması sonucunda elde edilen ve en iyi ABEP değerine sahip eşleştirme noktaları optimum alt-blok seti olarak belirlenir $\left(\boldsymbol{S}^{\text {optimum }}\right)$. 


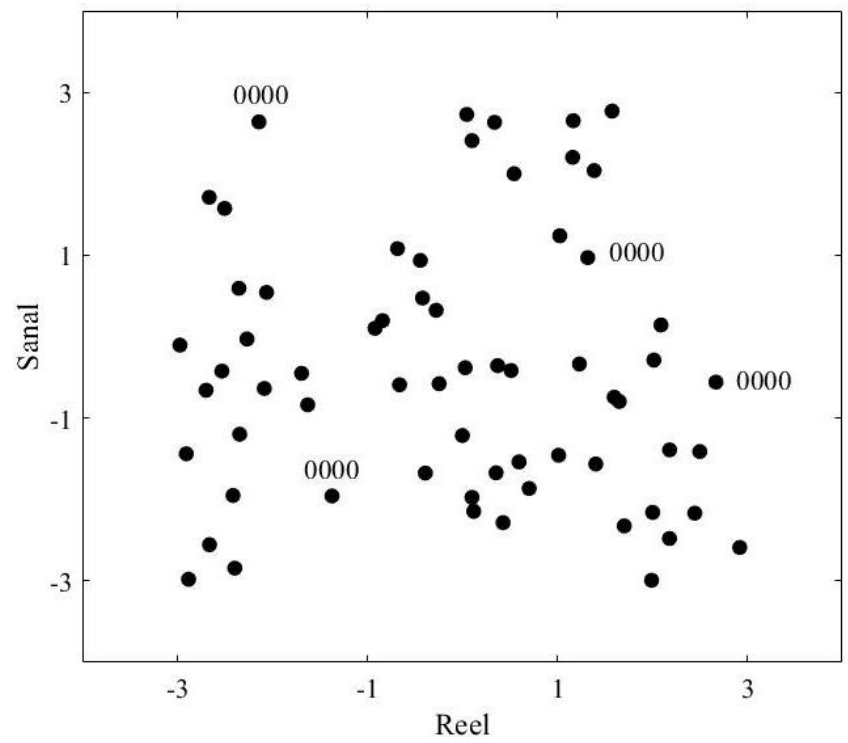

Şekil 5. KKO Algoritması ile elde edilen alt-blok seti örneği.

$\boldsymbol{S}^{\text {optimum }}=\left[\begin{array}{llll}\boldsymbol{S}^{(1)} & \boldsymbol{S}^{(2)} & \cdots & \boldsymbol{S}^{\left(2^{p}\right)}\end{array}\right]$

burada $\boldsymbol{S}^{(i)}$ her bir alt-bloğu, $i$ ise alt-blok indeksini temsil etmektedir. Şekil 5'de KKO algoritması sonucu elde edilmiş bir alt-blok seti için örnek eşleştirme noktaları görülmektedir. Şekil 5'de ' $0000^{\prime}$ veri bit dizisi için eşleştirme noktaları ayrıca gösterilmektedir.

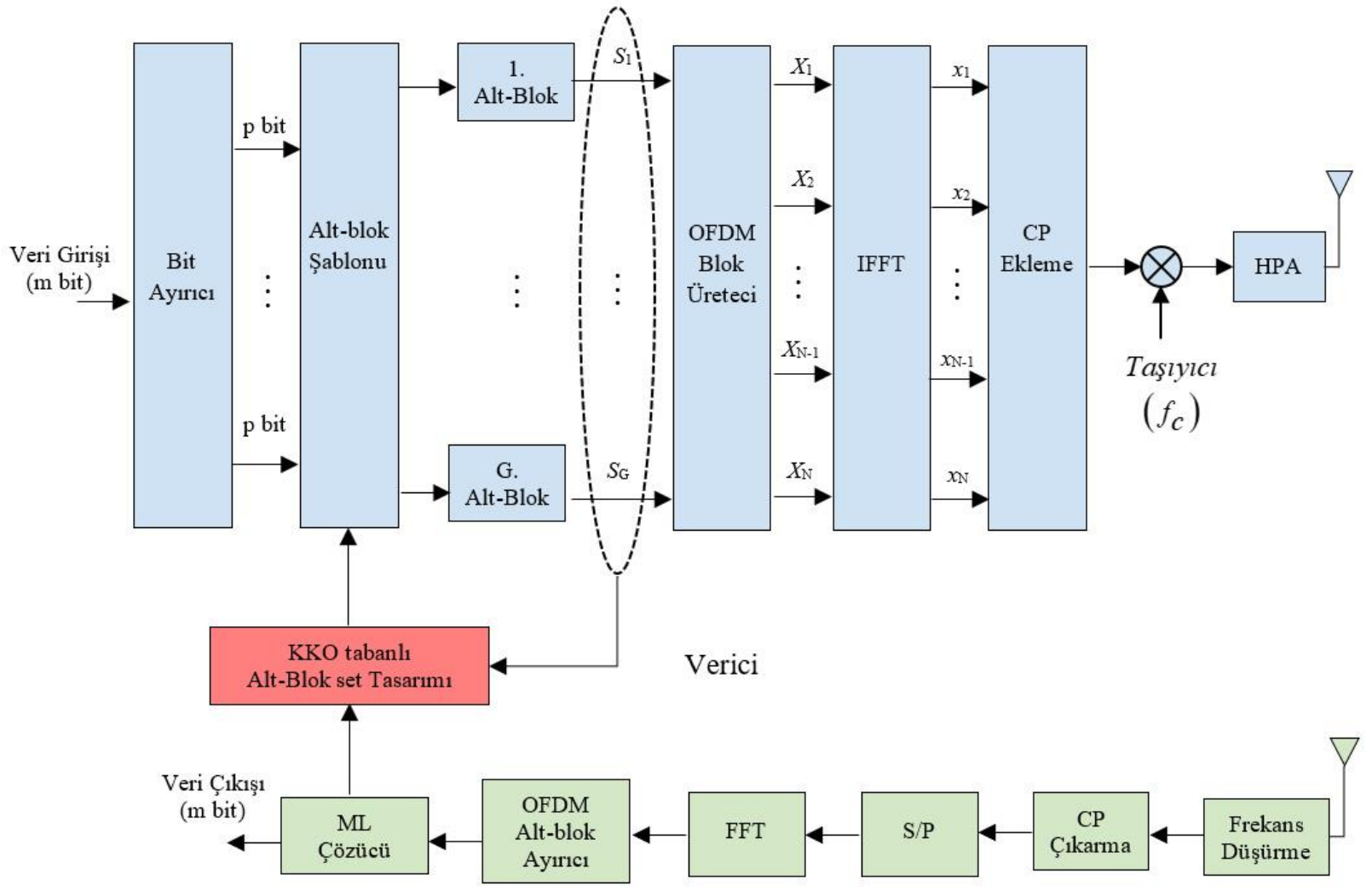

Alic1

Şekil 6. KKO-OFDM-AIM iletim tekniğinin blok şeması.

Optimizasyon, PSK/QAM takımyıldızı uzayında alt-taşıyıcı noktalarının rasgele belirlenmesiyle başlar. $c$ adet üretilen alt-blok setlerinin uygunluk değerleri, sistemin ABEP başarısına göre belirlendikten sonra alt-blok setleri uygunluk değerlerine göre en iyiden en kötüye göre sıralanır. İlerleyen çevrimlerdeki yeni alt-taşıyıcı noktalarının konumu, önceki çevrimlerde elde edilen çözümlerin 
uygunluk değerleri bilgileri dikkate alınarak belirlenmektedir. OFDM-AIM'deki aynı eşleştirme noktası $n$ kez çoğullanırken, KKOOFDM-AIM tarafından çeşitlilik kazancının artırılması amacıyla $n$ farklı nokta olarak belirlenir. Böylelikle KKO algoritması, $n_{S_{g}} * n$ eşleştirme noktası için optimizasyon gerçekleştirmektedir. KKO-OFDM-AIM tekniği blok şeması Şekil 6'da gösterilmektedir. Şekil 6'da görüleceği üzere KKO-OFDM-AIM'in alt-blok oluşturma yapısı OFDM-AIM'den farklıllık gösterirken diğer alt işlemler benzerlik göstermektedir.

\section{Simülasyon Sonuçları}

Bu bölümde, önerilen KKO-OFDM-AIM tekniğinin performansı ABEP ve BER başarı kriterlerine göre değerlendirilmiştir. KKOOFDM-AIM tekniğinin performans1, Tablo 3'de verilmiş olan sistem parametrelerine göre OFDM, OFDM-IM, CI-OFDM-IM, OFDMAIM ve GA-OFDM-AIM teknikleri ile $L_{c h}=10$ uzunluğundaki frekans seçici Rayleigh sönümlemeli kanal için karşılaştırılmıştır.

Tablo 3. Sistem parametreleri

\begin{tabular}{cc}
\hline Parametre & Değer \\
\hline Toplam Alt-taşıyıcı & $N=G * n=128$ \\
Alt-blok & $G=32$ \\
Bir alt-bloktaki alt-taşıyıcı sayısı & $n=4$ \\
Bir sembolde iletilen bit sayısı & $m=G * p=128, \quad p=4$ için \\
Bir alt-bloktaki aktif alt-taşıyıcı sayısı & $m=G * p=256, \quad p=8$ için \\
(KKO-OFDM-AIM / OFDM-IM) & $k=4 / k=2$ \\
KKO için araştırma sayısı & $A S=K S *$ İterasyon \\
& $=10 * 100=1000$ \\
Döngüsel önek (CP) & $L_{C P}=N / 8=16$ \\
Rayleigh Kanal için kanal uzunluğu & $L_{c h}=10$ \\
Modülasyon & $16 \mathrm{QAM}, p=4$ için \\
& $256 \mathrm{QAM}, p=8$ için \\
Spektral verimlilik (SV) & $S V=0.8889[\mathrm{bit} / \mathrm{s} / \mathrm{Hz}], \mathrm{m}=128$ \\
& $S V=1.7778[\mathrm{bit} / \mathrm{s} / \mathrm{Hz}], \mathrm{m}=256$
\end{tabular}

Bir KKO-OFDM-AIM sembolü, $p$ veri bitlerine göre alt-blok setinden seçilen alt-bloklardan oluşmaktadır. $p=4$ bit ve $p=8$ bit veri dizisi uzunlukları için bir alt-blok setinde sırasıyla, $n_{S_{g}}=16$ ve $n_{S_{g}}=256$ alt-blok bulunmaktadır.

KKO algoritması ile optimum alt-blok seti tasarımında popülasyon büyüklüğ̈̈ (karınca sayıs1) $K S=100$, iterasyon sayıs1 İterasyon = 125 olarak belirlenmiştir. Önerilen KKO-OFDM-AIM ile ABEP ve BER performanslarının karşılaştırıldığı GA-OFDMAIM tekniğinde genetik algoritma, popülasyon büyüklüğü Pop $=100$ ve iterasyon sayısı İterasyon $=125$ olarak araştırmasını gerçekleştirmiştir. Böylece KKO ve genetik algoritma, $A S=12500$ araştırma sayısı için karşılaştırılmıştır.

Şekil 7'de KKO-OFDM-AIM ve GA-OFDM-AIM tekniklerinin ABEP performansları $S E=0.8889[\mathrm{bit} / \mathrm{s} / \mathrm{Hz}]$ spektral verimlilik değeri için karşılaştırılmıştır. Böylece karşılaştırma, her iki teknik için de $p=4$ ve $m=128$ değerleri için gerçekleştirilmiştir. Şekil 7'de görüleceği üzere önerilen KKO-OFDM-AIM, GA-OFDM-AIM tekniğine göre neredeyse tüm iterasyon sayısı değerlerinde daha düşük bir ABEP değerine sahiptir. Bu durumda KKO algoritması sonucu elde edilen alt-blok setinin uygunluğunun, genetik algoritma sonucu elde edilen alt-blok setinin uygunluğundan daha yüksek olduğu anlaşılmaktadır. 


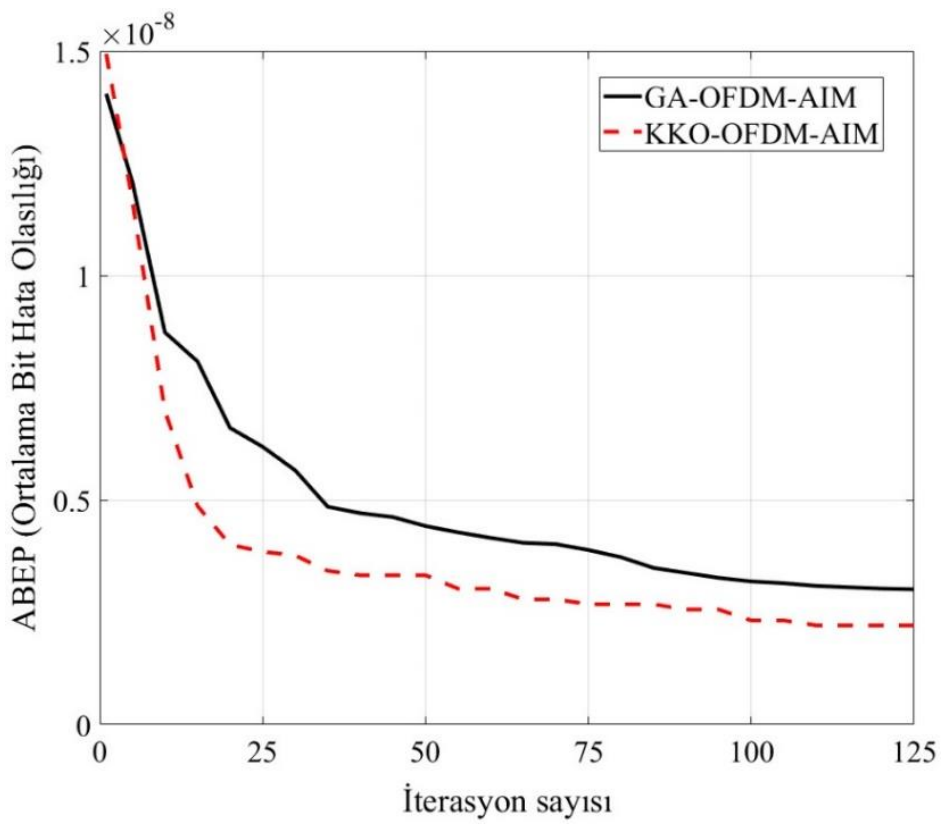

Şekil 7. KKO-OFDM-AIM ve GA-OFDM-AIM tekniklerinin, uygunluk fonksiyonu olarak ABEP karşılaştırmaları.

Şekil 8 ve 9'da OFDM (Hwang vd., 2009), OFDM-IM (Başar vd., 2013), CI-OFDM (Başar, 2015), OFDM-AIM (Shi vd., 2019), GAOFDM-AIM (Shi vd., 2019) ve önerilen KKO-OFDM-AIM İletim tekniklerinin SNR-BER performansları karşılaştırmaları görülmektedir. Şekil 8 ve 9'da karşılaştırmalar sırasıyla, $\mathrm{SE}=0.8889 \mathrm{bits} / \mathrm{s} / \mathrm{Hz}$ ( $p=4$ bit $)$ ve $\mathrm{SE}=1.7778$ ( $p=8$ bit $)$ spektral verimlilik durumları için gerçekleştirilmiştir. Her iki şekilden de görüleceği gibi önerilen KKO-OFDM-AIM, en başarılı BER performansına sahip iletim tekniği olmuştur. Örneğin Şekil 9 dikkate alındığında, KKO-OFDM-AIM, BER $=10^{-5}$ değerinde GAOFDM, CI-OFDM-IM ve OFDM-AIM tekniklerinden sırasıyla, $1 \mathrm{~dB}, 7.5 \mathrm{~dB}$ ve $8 \mathrm{~dB}$ daha iyi bir SNR (işaret gürültü oranı, Signal to Noise Ratio) değerine sahip olduğu görülmektedir.

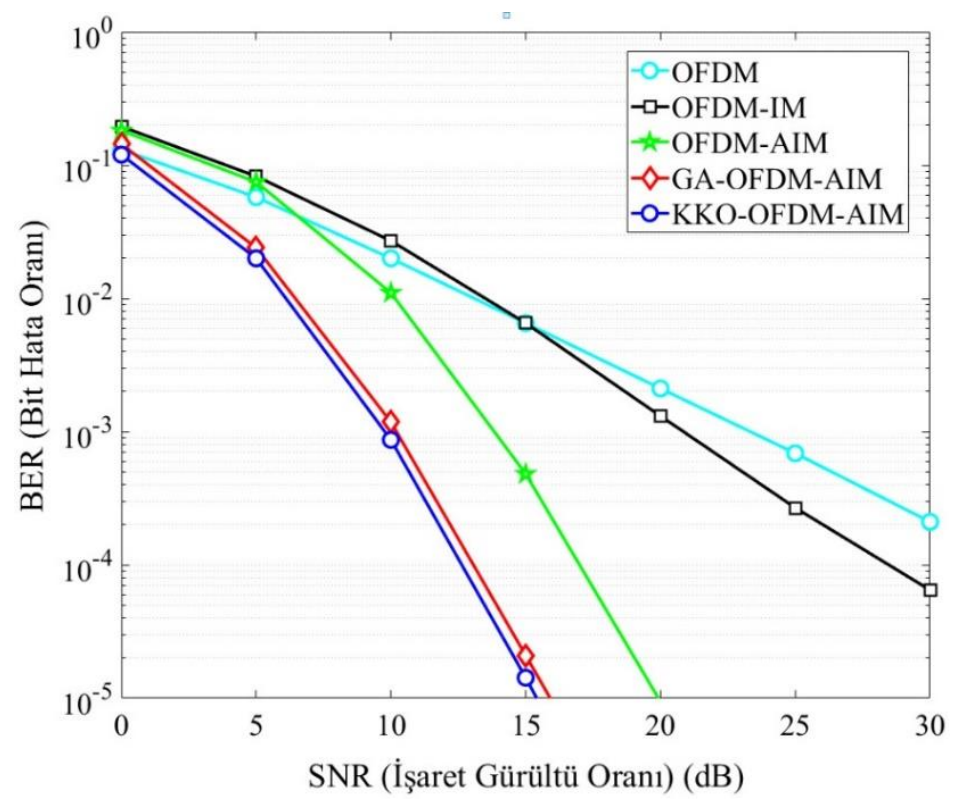

Şekil 8. Önerilen KKO-OFDM-AIM ile diğer iletim tekniklerinin SNR-BER performansı karşılaştırılması (SE=0.8889 bits/s/Hz). 


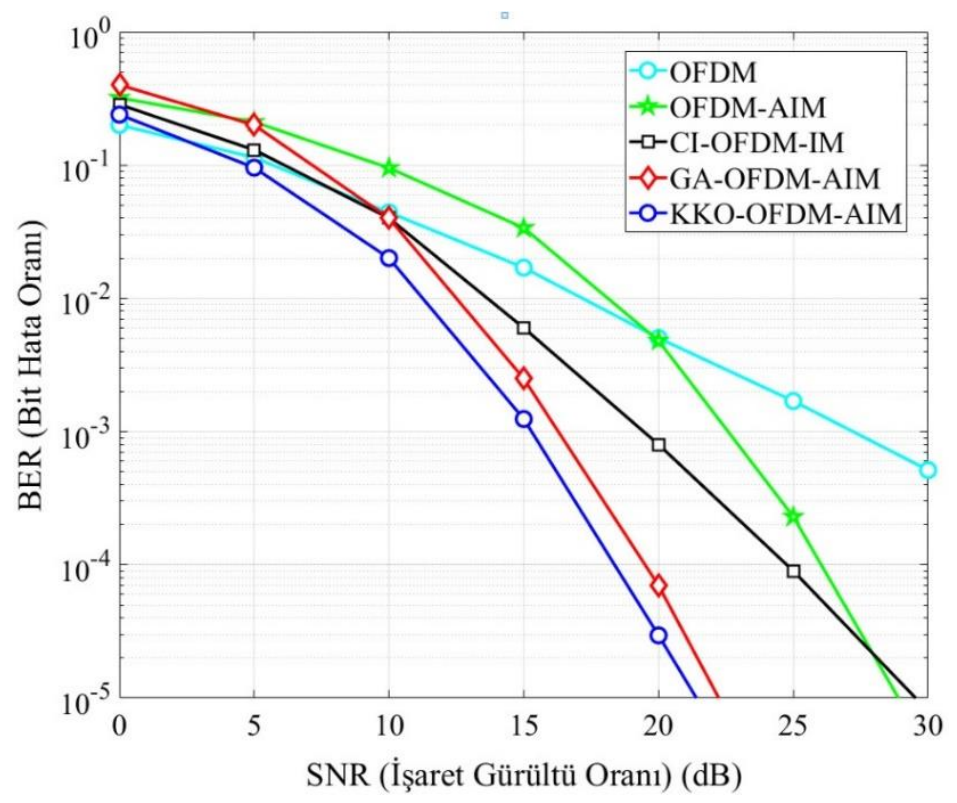

Şekil 9. Önerilen KKO-OFDM-AIM ile diğer iletim tekniklerinin SNR-BER performansı karşılaştırılması (SE=1.7778 bits/s/Hz).

\section{Sonuçlar ve Gelecek Çalışmalar}

OFDM-IM (İndeks Modülasyonlu Dikgen Frekans Bölmeli Çoğullama), sahip olduğu yüksek enerji ve spektral verimliliğinden dolay gelecek nesil kablosuz ağlar için aday iletim teknikleri arasında yer almaktadır. Son yıllarda OFDM-IM tekniğinin geliştirilmesi amacıyla literatürde birçok teknik önerilmiştir. Bu tekniklerden birisi olan OFDM-AIM (Tüm İndeks Modülasyonlu OFDM)'de, veri iletimi için kullanılmayan alt-taşıyıcılara sahip olan OFDM-IM'den farklı olarak tüm alt-taşıyıcılar aktif duruma getirilmiş ve bu yolla spektral verimliliğin daha da artırılması amaçlanmıştır. Bu makalede, OFDM-AIM'in bit hata oranı (BHO) performansının iyileştirilmesi amacıyla karınca kolonisi optimizasyonu (KKO) algoritması tabanlı bir alt-blok set tasarımı önerilmiştir (KKO-OFDMAIM). Klasik OFDM-AIM tekniğinde alt-blok seti, PSK/QAM takımyıldızı noktalarından oluşurken; önerilen KKO-OFDM-AIM tekniğinde, PSK/QAM takımyıldızı uzayının tamamında en yüksek uygunluk değerine (en düşük ABEP değeri) sahip alt-blok seti araştırılır. Simülasyon çalışmalarında, KKO-OFDM-AIM'in ABEP performansı, bir başka sezgisel optimizasyon yöntemi olan genetik algoritma (GA) tabanlı OFDM-AIM (GA-OFDM-AIM) ile karşılaştırılmış ve daha düşük ABEP değerlerine sahip olduğu görülmüştür. KKO-OFDM-AIM'in BHO performansı ise OFDM, OFDM-IM, OFDM-AIM ve GA-OFDM-AIM teknikleri ile karşılaştırılmış ve en iyi BHO başarısına sahip olduğu görülmüştür.

Gelecek çalışmalar temel olarak iki yönde düşünülebilir. Birincisi, alt-blok set tasarımı için önerilen KKO algoritması, DM-OFDMIM gibi diğer IM tabanlı iletim tekniklerinin BHO performanslarının artırılması için de uygulanabilir. İkinci olarak, KKO algoritması veya başka sezgisel algoritmalar IM tabanlı tekniklerin farklı problemleri için kullanılabilir. Örneğin alt-taşıyıcı sayısı modülasyonu tabanlı OFDM (SNM-OFDM) tekniğindeki (Dang vd., 2019) en uygun alt-taşıyıcı sayısının araştırılması için sezgisel algoritmalar kullanılabilir.

\section{Referanslar}

Andrews, J. G., Ghosh, A., \& Muhamed, R. (2007). Fundamentals of WiMAX: understanding broadband wireless networking (1st ed.). Pearson Education.

Andrews, J. G., Buzzi, S., Choi, W., Hanly, S. V., Lozano A., Soong, C. K., \& Zhang, J.C. (2014). What Will 5G Be?. IEEE Journal on Selected Areas in Communications, 32(6), 1065-1082. doi: 10.1109/JSAC.2014.2328098

Başar, E., Aygölü, Ü., Panayırc1, E., \&Poor, H. V. (2013). Orthogonal Frequency Division Multiplexing With Index Modulation. IEEE Transactions on Signal Processing, 61(22), 5536-5549. doi: 10.1109/TSP.2013.2279771

Basar, E. (2016). On Multiple-Input Multiple-Output OFDM with Index Modulation for Next Generation Wireless Networks. IEEE Transactions on Signal Processing, 64( 15), 3868-3878. doi: 10.1109/TSP.2016.2551687

Başar, E. (2015). OFDM With Index Modulation Using Coordinate Interleaving. IEEE Wireless Communications Letters, 4(4), 381 384. doi: 10.1109/LWC.2015.2423282 
Boroujeny, B. F., \& Moradi, H. (2016). OFDM Inspired Waveforms for 5G. IEEE Communications Surveys \& Tutorials, 18(4), $2474-$ 2492. doi: $10.1109 /$ COMST.2016.2565566

Chen, W., \& Zhang, J. (2013). Ant colony optimization for software project scheduling and staffing with an event-based scheduler. IEEE Transactions on Software Engineering, 39(1), 1-17. doi: 10.1109/TSE.2012.17

Crow, B. P., Widjaja, I., Kim, J. G., \& Sakai, P. T. (1997). IEEE 802.11 wireless local area networks. IEEE Communications Magazine, 35(9), 116-126. doi: 10.1109/35.620533

Dahlman, E., Parkvall, S., \& Skold, J. (2013) 4G: LTE/LTE-advanced for mobile broadband (2nd ed.). Academic press.

Dang, S., Ma, G., Shihada, B., \& Alouini, M. S. (2019). Enhanced orthogonal frequency-division multiplexing with subcarrier number modulation. IEEE Internet Things Journal, 6(5), 7907-7920. doi 10.1109/JIOT.2019.2914175

Fan, R., Yu, Y. J. \& Guan, Y. L. (2015). Generalization of Orthogonal Frequency Division Multiplexing With Index Modulation. IEEE Transactions on Wireless Communications, 14(10), 5350-5359. doi: 10.1109/TWC.2015.2436925

Hwang, T., Yang, C., Wu, G., Li, S., \& Li, G.Y. (2009). OFDM and Its Wireless Applications: A Survey. IEEE Transactions on Vehicular Technology, 58(4), 1673-1694. doi: 10.1109/TVT.2008.2004555

Lu, L., Li, G. Y., Swindlehurst, A. L., Ashikhmin, A., \& Zhang, R. (2014). An Overview of Massive MIMO: Benefits and Challenges. IEEE Journal of Selected Topics in Signal Processing, 8(5), 742-758. doi: 10.1109/JSTSP.2014.2317671

Mao, T., Wang, Z., Wang, Q., Chen, S., \& Hanzo, L. (2017). Dual-Mode Index Modulation Aided OFDM. IEEE Access, 5, 50-60. doi: 10.1109/ACCESS.2016.2601648

Shi, Y., Lu, X., Gao, K., Zhu, J., \& Wang, S. (2019). Subblocks Set Design Aided Orthogonal Frequency Division Multiplexing With All Index Modulation. IEEE Access, 7, 52659-52668. doi: 10.1109/ACCESS.2019.2909911

Shi, Y., Lu, X., Gao, K., Zhu, J., \& Wang, S. (2019). Genetic Algorithm Aided OFDM With All Index Modulation. Communications Letters, 23(12), 2192-2195. doi: 10.1109/LCOMM.2019.2942915

Wen, M., Zheng, B., Kim, K. J., Di Renzo, M., Tsiftsis, T. A., Chen, K., \& Al-Dhair, N. (2019). A Survey on Spatial Modulation in Emerging Wireless Systems: Research Progresses and Applications. IEEE Journal on Selected Areas in Communications, 37(9), 19491972. doi: 10.1109/JSAC.2019.2929453

Wen, M., Ye, B., Basar, E., Li, Q., \& Ji, F. (2017). Enhanced Orthogonal Frequency Division Multiplexing With Index Modulation. IEEE Transactions on Wireless Communications, 16(7), 4786-4801. doi: 10.1109/TWC.2017.2702618

Xiao, Y., Wang, S., Dan, L., Lei, X., Yang, P., \& Xiang, W. (2014). OFDM With Interleaved Subcarrier-Index Modulation. IEEE Communications Letters, 18(8), 1447-1450. doi: 10.1109/LCOMM.2014.2332340

Yıldırım, M. (2020). ABC Algorithm-Based Subblocks Set Design for OFDM With All Index Modulation. IEEE Communications Letters, 24(12), 2926-2930. doi: 10.1109/LCOMM.2020.3019476 